\title{
Estructura morfológica y recuperación léxica ${ }^{1-2}$
}

\author{
Susana del Viso \\ Universidad de Oviedo \\ José E. García-Albea
}

Universidad Complutense de Madrid

\section{INTRODUCCION}

En el marco de la Psicolingüística, se ha venido dando un creciente interés por el estudio del componente léxico, interés que se ha puesto de manifiesto en numerosas in: vestigaciones a lo largo de los últimos años. Lo que finalmente tratan de determinar estos trabajos es cómo se representa y organiza la información de que dispone el hablante-oyente acerca de las palabras de su idioma y, por otro lado, cuáles son las estrategias que utiliza para acceder a dicha información.

Numerosas investigaciones, realizadas en su mayor parte en inglés, parecen haber demostrado que las entradas léxicas no se corresponden puntualmente con las palabras, sino que se trata de representaciones más abstractas. Uno de los aspectos claves en.el estudio de la organización del léxico interno se refiere justamente al formato de las representaciones y a las recodificaciones que han de llevarse a cabo a partir del input sensorial para que el acceso tenga lugar. En la actualidad, parece probado que al reconocer un input verbal - ya sea visual o acústico-, el sujeto lleva a cabo un análisis preliminar, centrándose en la primera porción del estímulo para acceder al léxico. Aunque todavía no está claro en qué consiste esa primera porción (véase Taft, 1979a; García-Albea, 1980, 1984), sí parece demostrado que el análisis de ese primer segmento es suficiente para acceder a la representación mental correspondiente. Prueba de ello son los experimentos realizados por $\mathrm{Taft}$ (1979b) y Taft y Forster (1975, 1976), a partir de los cuales los autores concluyen que las entradas léxicas consistirían en una representación de la raíz de las palabras, a la cual se añadirían los elementos afijos a través de una serie de reglas. Taft y Forster (1975) llegaron a proponer un modelo de reconocimiento de palabras que incorpora este proceso de segmentación del input. (Puede verse una revisión detallada de este tema, así como el esquema del modelo propuesto en del Viso y García-Albea, 1985.)

Así, pues, según estos autores, la unidad de acceso sería el morfema. Esta idea tiene además claras implicaciones con respecto a

1 Esta investigación ha sido posible gracias a una Ayuda de Investigación Cooperativa del Comité Conjunto Hispano-Norteamericano para Asuntos Educativos y Culturales.

2 Parte de esta investigación se presentó en el Symposium sobre Actividad Humana y Procesos Cognitivos (Madrid, 19 al 22 de diciembre de 1984). 


\section{Estudios}

la cuestión de la organización del léxico interno y del formato de las representaciones. Se postula que éstas vendrían a equivaler a la raíz de las palabras, lo cual implica que en una misma entrada léxica estarían representadas las distintas palabras pertenecientes a una misma familia (por ej., jabón, jabonera, enjabonar, etc.)

Esta hipótesis ha recibido un considerable apoyo experimental en los distintos campos de estudios del lenguaje: comprensión, producción, adquisición y patología. No obstante, no está exenta de controversia, ya que otros autores (por ej., Holyoak et al., 1976; Manelis y Tharp, 1977; Rubin, Becker y Freeman, 1979) han propuesto, a partir de sus resultados experimentales, que cada palabra posee su propia entrada léxica separada de las de otras palabras relacionadas. Si bien Taft $(1979 \mathrm{~b}, 1981)$ ha respondido a los puntos de divergencia que estos resultados plantean en relación con su modelo, no queremos pasar por alto el hecho de que existen posturas controvertidas con respecto al tema que nos ocupa. (Para una revisión de la investigación en las distintas áreas psicolingüísticas, así como de la polémica entre los dos puntos de vista, véase del Viso y García-Albea, 1985.)

El trabajo que vamos a presentar pretende ofrecer nuevos datos experimentales sobre la cuestión de la representación léxica. Centrándonos en el área de la comprensión, hemos estudiado el reconocimiento de palabras aisladas bajo las dos modalidades sensoriales, visual y auditiva. Nuestros experimentos se dirigen fundamentalmente a esclarecer la función que cumple la estructura morfológica en el proceso de reconocimiento de palabras y, por tanto, en los mecanismos que rigen su recuperación. Más en concreto, nos planteamos los siguientes objetivos: 1) determinar la forma de representación léxica de las palabras y, más específicamente, las relaciones entre la representación de la forma base de una palabra y las formas relacionadas morfológicamente; 2) precisar qué tipo de estrategias emplean los hablantes para dar con las entradas léxicas; 3) comprobar si se obtienen los mismos resultados cuando los estímulos se presentan visual y auditivamente; y 4) efectuar una comparación translingüística entre los resultados obtenidos en inglés y los que se obtengan en castellano.

En los tres experimentos se utilizaron palabras con relaciones derivativas. Todos ellos están basados en el fenómeno de "priming» o facilitación por presentación previa (Meyer y Schvaneveldt, 1971; Forbach et al., 1974; Fischler, 1977; Scarborough et al., 1977; Blank y Foss, 1978; Forster, 1981; Carroll y Kirsner, 1982; Foss, 1982). En pocas palabras, el paradigma básico del «priming» consiste en presentar un estímulo determinado y, tras un cierto intervalo temporal (durante el cual pueden o no aparecer otros estímulos), presentarlo de nuevo para que sea reconocido por el sujeto. El resultado que se observa es que en la segunda presentación del estímulo en cuestión («target»), aunque esté separada temporalmente de la primera («prime»), se produce una notable disminución del tiempo empleado en reconocerlo. A partir de este paradigma, se han llevado a cabo distintas modificaciones; la principal consiste en que el «prime» y el «target» no sean exactamente el mismo estímulo, sino estímulos distintos, aunque relacionados de uno u otro modo. Los dos tipos de relación entre «prime» y «target» más estudiados son las relaciones semánticas y las morfológicas (Stanners, Neiser, Hernon y Hall, 1979; Stanners, Neiser y Painton, 1979).

Específicamente, el «priming» semántico consiste en presentar sucesivamente dos palabras relacionadas semánticamente (por ej., doctor-enfermera), ante cada una de las cuales el sujeto debe realizar una tarea de decisión léxica. La latencia de decisión para la palabra enfermera es menor si ha sido precedida por doctor que si lo fue por una palabra no relacionada. Los experimentos de Meyer y su equipo (Meyer y Schvaneveldt, 1971; Meyer, Schvaneveldt y Ruddy, 1972; Schvaneveldt y Meyer, 1973), de los primeros realizados en este área, encontraron efectos de "priming» semántico en la memoria utilizando la tarea de decisión léxica. Sus resultados indican que las decisiones de los sujetos son más rápidas para las palabras que han sido precedidas por un «prime» que para las que no lo han sido. 
También se ha demostrado (por ej., Blank y Foss, 1978) que el procesamiento de una palabra dentro de una oración es más rápido si dicha palabra ha sido precedida por otra relacionada semánticamente.

Más relacionado con nuestra investigación es el trabajo de Stanners y sus colaboradores. Estos autores realizaron cuatro experimentos para investigar el estatus de memoria de las formas inflexivas de los verbos $(-s,-e d,-i n g)$, los pasados irregulares y los adjetivos y nombres derivados de verbos, utilizando el paradigma de «priming» con una tarea de decisión léxica. La cuestión central era ver si estas formas flexivas y derivativas tenían representaciones de memoria independientes de sus respectivos verbos base. La aplicación de esta técnica implicaba la comparación del tiempo de reacción (TR), o lacencia de decisión, empleado en responder a un verbo base cuando había sido precedido por él mismo con el TR empleado en responder ante ese mismo verbo habiendo sido precedido por una de sus variaciones flexivas o derivadas. Por ejemplo, en el caso de las inflexiones, si existieran representaciones de memoria independientes para lend y para -ing (pero no para lending), al presentarse la palabra lending, el sujeto tendría que acudir a la representación de lend. Por tanto, el efecto de «priming» de lending sobre lend debería ser aproximadamente de la misma magnitud que el de lend sobre lend.

Así, pues, ésta parece ser una tẹcnica sensible y adecuada para el propósito que nos ocupa. Además, posee una ventaja adicional, ya que «con el contrabalanceo apropiado, puede responderse a las principales cuestiones experimentales comparando los TR exactamente de las mismas palabras bajo distintas condiciones de "priming», eliminando así los problemas que surgen cuando se pretende emparejar variables tales como la frecuencia de uso o la longitud» (Stanners, Neiser, Hernon y Hall, 1979, pág. 401).

\section{EXPERIMENTO I}

En este experimento, se utilizó un conjunto de nombres base (no derivados), cada uno de los cuales daba lugar, por derivación, a otro nombre, un verbo y un adjetivo (por ej., flor-florero-florecer-florido).

La hipótesis de trabajo era la siguiente: suponiendo que el reconocimiento de una palabra se ve facilitado por la presentación previa de la misma, se trata de ver si también se dan efectos de facilitación, y en qué medida, cuando lo que se presenta en primer lugar es una palabra derivada de la que va a aparecer a continuación.

A partir de aquí, se formulan las siguientes predicciones específicas:

1. Al presentar una palabra precedida por ella misma (por ej., flor... flor), el TR disminuirá de la primera presentación a la segunda.

2. ${ }^{a}$ Suponiendo que para reconocer una palabra derivada, la estrategia a seguir sea la de descomponerla morfológicamente en raíz + afijos y centrarse en la raíz para acceder al léxico, el reconocimiento de florero implicaría la descomposición flor-ero y el acceso a la entrada «flor». En este sentido, la segunda predicción sería que el reconocimiento de una palabra base se verá facilitado (el TR será menor) por la presentación previa de una palabra derivada de ella.

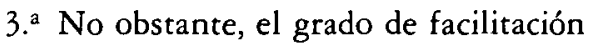
de una palabra sobre sí misma siempre será mayor que el de una forma derivada sobre su base correspondiente, debido principalmente al hecho de que, en el primer caso («prime» y «target» idénticos), una vez que el sujeto ha dado con la entrada léxica, no tiene que efectuar operaciones adicionales para verificar la concordancia entre raíz y sufijo.

4. ${ }^{a}$ Los efectos de facilitación producidos sobre la palabra base por las distintas formas derivadas serán equivalentes, independientemente de la categoría gramatical de las derivadas (nombre, verbo, adjetivo).

Según la hipótesis de que las palabras derivadas poseen entradas léxicas independientes de la de su forma base, no se cumplirían estas predicciones, salvo la $1 .^{\mathrm{a}}$. En el caso de la 2. ${ }^{a}$, florero no facilitaría el reconocimiento de flor, ya que no habria que acudir a, o pasar por la entrada «flor» para reconocer la derivada. Las predicciones $3 .^{\mathrm{a}}$ 
y $4 .^{a}$ no tendrían sentido, una vez establecido lo anterior.

\section{METODO}

Sujetos. Participaron 72 sujetos ( 20 hombres y 52 mujeres) estudiantes de 5.9 de Psicología en la Universidad Complutense de Madrid. Fueron seleccionados aleatoriamente, con la única condición de que fueran monolingües. Los 72 sujetos se distribuyeron en tres grupos iguales y, a su vez, cada grupo se dividió en dos subgrupos de 12 sujetos. En los tres grupos se presentó la misma secuencia de estímulos, aunque alternando las distintas condiciones experimentales entre grupos y subgrupos, tal como se describe en el siguiente apartado.

Materiales y Diseño. A cada sujeto se le presentaban 192 estímulos (96 palabras y 96 no-palabras). Se partió de una lista original de 36 palabras (nombres primitivos en singular) que pudieran dar lugar a tres formas derivadas: otro nombre, un verbo y un adjetivo.

Se establecieron dos condiciones experimentales: una "condición crítica» y una «condición de repetición». En la primera, cada una de las palabras base se presentaba precedida por una de sus formas derivadas; es decir, el «prime» lo constituía una palabra derivada y el «target», la palabra base corkespondiente. En la condición de repetición, la palabra base se presentaba precedida por ella misma; en este caso, pues, el «prime» y el «target» eran exactamente la misma palabra.

Para hacer esto posible, la lista original de 36 palabras se dividió aleatoriamente en tres conjuntos de 12 palabras, en cada uno de los cuales se hicieron dos subconjuntos de 6. Al hacer estas divisiones, se tuvo en cuenta que dentro de cada conjunto y de cada subconjunto quedara el mismo número de palabras con el mismo número de letras. A cada uno de los tres conjuntos se le asignó una categoría gramatical distinta (nombre/verbo/adjetivo) que variaba de un grupo de sujetos a otro, y a cada uno de los dos subconjuntos, una condición experimental distinta (crítica/repetición), que va- riaba de un subgrupo a otro de sujetos. Así, pues, la lista original de 36 palabras daba lugar a 72 estímulos. A éstos se añadieron 24 palabras más (entre las que se incluían formas base y derivadas independientes unas de otras), que no se presentaban precedidas ni por ellas mismas ni por formas relacionadas. Estas 24 palabras «de relleno» sirvieron para organizar la secuencia de estímulos y camuflar de algún modo las repeticiones y cuasi-repeticiones de los estímulos propiamente experimentales, que se producían en intervalos de 7 a 12 estímulos.

Se determinó que las palabras utilizadas no fuesen ambiguas ni en cuanto a su pertenencia a una categoría gramatical ni en cuanto a su significado. Además, los nombres se presentaban siempre en singular; los adjetivos, en masculino singular; y los verbos, en infinitivo.

La construcción de las no-palabras se guió por los mismos criterios formales y estructurales que se habían seguido en el caso de las palabras (apariencia de nombre, verbo o adjetivo y de forma primitiva o derivada), presentándose asimismo según las condiciones experimentales mencionadas.

Los estímulos utilizados se presentan en el Apéndice I.

Los 192 estímulos que veía cada sujeto se presentaron distribuidos en cuatro bloques experimentales de 48 elementos cada uno. Dentro de cada bloque, los estímulos aparecían en un orden cuasi-aleatorio, con la restricción de que no apareciesen más de cuatro items consecutivos del mismo tipo de respuesta (palabra/no-palabra), de la misma condición experimental (crítica/repetición) ni del mismo intervalo entre «prime»y «target» $(7 / 8 / 9 / 10 / 11 / 12)$. Todas estas condiciones estaban igualmente representadas en los cuatro bloques.

La presentación de los bloques se hizo según un orden aleatorio entre los grupos y los subgrupos de sujetos, viendo cada uno de ellos todos los tipos de estímulos, sólo que bajo condiciones experimentales diferentes.

Antes de comenzar la tarea experimental, se presentó un bloque de práctica con 32 estímulos del mismo tipo que los experimentales. 
Procedimiento. Cada estímulo se presentaba en una diapositiva, donde aparecían las letras minúsculas en blanco sobre fondo negro. El intervalo interestímulos fue de 4 segundos, siendo el 1 segundo el tiempo de exposición de cada uno. Se utilizó una película de alto contraste (Kodak Eastman 5362) con el objeto de lograr una mayor nitidez de las letras con respecto al fondo.

Las diapositivas se proyectaban mediante una pantalla de transparencia mediante un proyector Kodak Ektagraphic (Modelo B-2AR) con bandeja de 80 diapositivas. La pantalla estaba colocada a unos 2 metros del proyector, situándose el sujeto al otro lado de la misma. Teniendo en cuenta que el sujeto se encontraba aproximadamente a 80 centímetros de la pantalla, el ángulo visual horizontal se estimó en $3,57 .^{\circ}$ y el vertical, en $0,72$. .

Se empleó un programador de tiempo de cuatro canales («Four Bank Timer»), que permitía programar la secuencia de estímulos con sus tiempos respectivos. Este aparato estaba conectado, por una parte, con el proyector, al que enviaba la orden de efectuar el cambio de diapositiva; por otra, con un adaptador de taquistoscopio que controlaba el tiempo de apertura del obturador adaptado al proyector; por último, con un medidor de TR en milisegundos. Este se ponía en funcionamiento en el momento mismo en que se producía la apertura del obturador; a su vez, estaba conectado con las llaves de respuesta situadas frente al sujeto. Al pulsar éste cualquiera de las dos llaves, se detenía el reloj, manteniéndose expuesto el TR unos 2 segundos para que el experimentador pudiera anotarlo. Según lo anterior, la latencia de respuesta se estimaba desde el momento de la aparición del estímulo. Además, el mismo sonido que se producía al pasar cada diapositiva servía como señal para que el sujeto estuviera preparado para recibir el siguiente estímulo.

La tarea del sujeto consistía en decidir si cada estímulo presentado era o no una palabra. Su decisión la expresaba pulsando una de las dos llaves de respuesta: la correspondiente al «SI» (con la mano preferida) para indicar que el estímulo era una palabra; la correspondiente al «NO» (con la otra mano), para indicar que no lo era. Las llaves de respuesta estaban conectadas con sendas luces de distinto color, a las que solamente tenía acceso el experimentador, a quien le permitían saber cuál había sido la respuesta del sujeto $y$, por tanto, saber si era correcta o no.

Al comienzo de la sesión experimental, se le leían las instrucciones al sujeto, explicándole en qué consistía su tarea.

Tratamiento de los datos. En el análisis de los datos, solamente se tuvieron en cuenta los TR correspondientes a las respuestas correctas. Los datos eliminados por constituir errores fueron el $2,3 \%$ de las respuestas a las palabras y el 2,7 \% para las no-palabras.

Por otra parte, se llevó a cabo una «limpieza» de datos según el siguiente criterio: un dato (TR de un sujeto determinado con respecto a un estímulo determinado) quedaba eliminado si su valor numérico excedía un valor crítico establecido en $X-1,5 s_{x}$, donde $X$ y $s_{x}$ se calculaban dós veces, una atendiendo a los TR obtenidos por ese sujeto en los distintos estímulos de un mismo tipo y otra atendiendo a los TR correspondientes a tal estímulo obtenidos por los distintos sujetos. Una «limpieza» de datos de este tipo no llega a eliminar más del $5 \%$ de los datos (concretamente el $2 \%$ para las palabras y el $2,3 \%$ para las no-palabras), reduciendo, en cambio, el posible «ruido» procedente de los TR extremos. De cara al análisis definitivo, todos los datos eliminados -bien por constituir errores, o bien por no haber pasado el filtro de «limpieza» anterior- se reemplazaban por el TR medio del sujeto en cuestión, calculándose posteriormente el TR medio correspondiente a cada estímulo.

A partir de los datos de las palabras, se calcularon en primer lugar los TR medios correspondientes a las distintas condiciones experimentales para cada una de las tres categorías gramaticales de las formas derivadas.

Se llevaron a cabo dos análisis: un análisis por estímulos y otro por sujetos. En ambos casos, se trataba de poner a prueba la significación de las diferencias entre los TR medios bajo las distintas condiciones experimentales. 
En el análisis por estimulos, el primer paso era comprobar el efecto de «priming» en la condición de repetición; es decir, comprobar si efectivamente se produce facilitación en el reconocimiento de una palabra por la presentación previa de la misma, ya que parte de nuestro análisis de datos se iba a basar en una modificación de este efecto. Para ello, se calculó la diferencia entre los TR medios del «prime» y del «target» de la condición de repetición, poniéndose a prueba la significación de la diferencia mediante el estadístico «t». El nivel de confianza mínimo para alcanzar la significación estadística se fijó en todos los casos en el $5 \%(\alpha=0,05)$.

La siguiente cuestión era comprobar si también se da un efecto facilitador cuando lo que precede a la forma base es una de sus derivadas. En este caso, la comparación oportuna se establece entre el «prime» de la condición de repetición y el «target» de la crítica. Por último, se determinó hasta qué punto eran equivalentes los efectos de facilitación producidos en uno y otro caso, para lo cual se comparó el «target» de la condición de repetición y el «target» de la crítica.

En el análisis por sujetos, se efectuaron las mismas comparaciones que en el anterior.

\section{RESULTADOS}

En las tablas 1 y 2 , se presentan los TR medios correspondientes a las distintas condiciones experimentales para cada una de las tres categorías gramaticales de las formas derivadas, a partir de los cuales se calcularon las diferencias oportunas.

Todas las diferencias de medias calculadas resultaron altamente significativas $(\mathrm{p}<$ $0,01)$ en ambos análisis.

Conviene mencionar el resultado adicional de que no se encontraron diferencias

\section{TABLA 1}

Tiempos de reacción medios correspondientes a las distintas condiciones experimentales en cada categoria gramatical (análisis por estimulos) $-E X P$. I-

\begin{tabular}{|c|c|c|c|}
\hline \multirow[b]{3}{*}{ Repetición } & \multicolumn{3}{|c|}{ Categoría gramatical } \\
\hline & Nombre & Verbo & Adjetivo \\
\hline & & & \\
\hline Prime .... & 581.08 & 581.64 & 582.06 \\
\hline Target .. & 527.19 & 532.56 & 526.58 \\
\hline \multicolumn{4}{|l|}{ Crítica } \\
\hline Prime & 680.92 & 663.58 & 673.33 \\
\hline 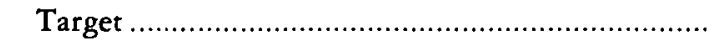 & 551.61 & 553.97 & 551.67 \\
\hline
\end{tabular}

TABLA 2

Tiempos de reacción medios correspondientes a las distintas condiciones experimentales en cada categoría gramatical (análisis por sujetos) -EXP. I-

\begin{tabular}{|c|c|c|c|}
\hline & \multicolumn{3}{|c|}{ Categoría gramatical } \\
\hline & Nombre & Verbo & Adjetivo \\
\hline Repetición & & & \\
\hline Prime.... & 581.93 & 583.78 & 584.78 \\
\hline Target .................... & 526.89 & 532.22 & 526.17 \\
\hline Crítica & & & \\
\hline Prime & 695.61 & 687.47 & 691.10 \\
\hline 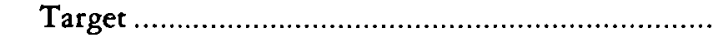 & 550.39 & 553.96 & 550.58 \\
\hline
\end{tabular}


significativas en el comportamiento de nombres, verbos y adjetivos. Asimismo, merece destacarse el hecho de que se llevaron a cabo las tres comparaciones relevantes para cada grupo de sujetos por separado, comprobándose que los tres grupos eran similares y comparables, ya que no hubo diferencias entre ellos, mostrando los tres el mismo patrón de resultados.

\section{DISCUSION}

En primer lugar, conviene subrayar la convergencia entre los resultados del análisis por estímulos y por sujetos.

Se comprueba la existencia de un fuerte efecto de repetición. Por otra parte, el efecto de facilitación que ejercen las palabras derivadas sobre la forma base correspondiente es muy notorio - si bien menor que el anterior-, lo cual viene a indicar que la representación de los nombres base se activó de alguna manera durante el proceso de reconocimiento de las derivadas. Una posibilidad es que el proceso de activación sea directo, en el sentido de que, al presentarse la derivada, se haya accedido a la representación del nombre base como si él mismo hubiera sido presentado. Posiblemente, al presentarse la derivada, se segmentan el nombre base y el sufijo antes de que comience la búsqueda en el léxico, accediendo posteriormente a la representación del nombre base.

Otra posibilidad es que la activación del nombre base sea un proceso indirecto del tipo que postulan Morton (1969), Collins y Loftus (1975), Meyer y Schvaneveldt (1976) $y$ otros autores, al proponer que el efecto de "priming» está basado en relaciones semánticas. La activación indirecta implica que el nombre base no es aislado del sufijo antes del acceso al léxico, sino que la representación de memoria de la palabra derivada se activa (directamente), lo cual, a su vez, activa (indirectamente) la representación del nombre base - con la cual está interconectada- Sin embargo, a este respecto, se puede argumentar que el efecto de facilitación encontrado en el caso del "priming» semántico, medido en milisegundos, es mu- cho menor que el encontrado por nosotros y otros investigadores (Stanners et al., 1979), en el caso del «priming» morfológico. Quizá esta diferencia pueda atribuirse al hecho de que las palabras que están relacionadas morfológicamente también lo están semánticamente. En cualquier caso, el efecto de facilitación debido a las relaciones morfológicas parece existir y podría calcularse restando del tiempo obtenido el que, por término medio, se atribuye a la facilitación semántica.

En general, los resultados que hemos obtenido parecen confirmar la idea de que presentar una palabra derivada facilita el posterior reconocimiento de la forma base. Estos resultados coinciden con los de Taft y Forster (1976). Ahora bien, este experimento no es definitivo ni responde a todas las cuestiones posibles. Aunque parece que los resultados apoyan el modelo propuesto por Taft y Forster, cabe preguntarse qué ocurriría si en lugar de presentar la forma derivada como «prime», fuera la base la que apareciera en primer lugar. Según las predicciones del modelo, parece que la forma derivada tendría que producir un efecto de facilitación sobre la base - como, de hecho, se ha encontrado- porque el acceso a la representación, por ejemplo, de florecer tiene que pasar por la descomposición morfológica y la utilización de la raíz flor. Sin embargo, no está muy claro qué ocurriría si flor precediera a florecer y hasta qué punto el efecto de facilitación sería de la misma mag. nitud que el anterior, o equivalente. En el modelo de acceso al léxico de Forster (1976), ya se hace alusión a las posibles «referencias cruzadas» o interconexiones que pueden darse entre distintas entradas léxicas dentro del «archivo principal» (o léxico interno).

Para explicar el efecto de «priming» del tipo doctor-enfermera, Forster afirma que no es necesario recurrir a la explicación de Meyer y Schvaneveldt $(1971,1976)$ en términos de «detectores» de palabras, y postula un sistema de referencias cruzadas entre las entradas léxicas del archivo principal. Una vez localizada la entrada «doctor» - cuyo acceso estaría controlado por la frecuencia de uso y, por tanto, tendría lugar a 
través de los archivos periféricos-, se establecería una transferencia a otras entradas relacionadas, como «enfermera» (a la cual no se accedería, en este caso, a través de los archivos periféricos).

En este sentido, cabe pensar que también existirán interconexiones entre entradas correspondientes a palabras relacionadas morfológicamente. Dado que en el experimento anterior no había ninguna condición experimental que nos permitiera poner a prueba este supuesto, diseñamos un nuevo experimento donde estuviese incluida.

\section{EXPERIMENTO II}

Este experimento constituye, pues, una versión modificada del anterior. La única variación que se introdujo fue la siguiente: en la condición crítica, en lugar de presentar la palabra base precedida por la derivada, se procedió al contrario. El principal objetivo de esta modificación era poder comparar el efecto de facilitación obtenido cuando la palabra derivada precede a la base (Experimento I) con el obtenido cuando es la palabra base la que precede a la derivada (Experimento II). Es decir, se trata de ver hasta qué punto la presentación previa de una palabra base facilita el reconocimiento de una de sus derivadas, que puede pertenecer o no a la misma categoría gramatical. $\mathrm{El}$ que hubiera o no diferencias entre estos dos efectos sería un dato relevante de cara a entender mejor la forma de representación léxica de las palabras y el papel que desempeñan las relaciones morfológicas entre ellas. Las posibles diferencias entre ambos efectos y el sentido o dirección de las mismas constituiría un apoyo para el tipo de representación común, centrado en la raíz y en la aplicación de reglas morfológicas para reconocer las derivadas. En cambio, la ausencia de diferencias indicaría que las representaciones de la forma base y de la derivada estarían de algún modo interconectadas, pero no se podría hablar de una dependencia unilateral (de la representación de la forma derivada con respecto a la de la base) a efectos de reconocimiento.

\section{METODO}

Sujetos. Participaron 72 sujetos de la misma procedencia que los anteriores, ninguno de los cuales había participado previamente en el Experimento I.

Materiales $y$ diseño. Fueron los mismos que en el experimento anterior, con la única diferencia que ya hemos mencionado, en el caso de la condición crítica.

Procedimiento. El mismo que en el Experimento I, sólo que esta vez, los estímulos se pasaron invirtiendo el orden de colocación de las diapositivas en la bandeja del proyector.

Tratamiento de los datos. Los TR medios para cada estímulo se calcularon por el mismo procedimiento anterior.

Conviene observar, antes de seguir adelante, que en este experimento no se iban a efectuar las tres comparaciones que se llevaron a cabo en el anterior, dado que se había concebido desde el principio como complementario de aquél y ya estaba previsto realizar las comparaciones a través de ambos. Así, pues, en primer lugar, se hallaron las diferencias entre el «prime» de la condición de repetición y el «target» de la crítica, ambas del Experimento I. (Recordemos que, en ambos casos, se trataba exactamente de la misma palabra - la forma base-, sólo que en el primer caso era la primera vez que aparecía, mientras que en el segundo, había sido precedida por una de sus formas derivadas.) Denominamos $\mathrm{X}_{1}$ a la primera de estas puntuaciones, $X_{2}$ a la segunda y $\mathrm{V}$ a la diferencia resultante.

En segundo lugar, se calcularon las diferencias entre el «prime» de la condición crítica del Experimento I y el «target» de la misma condición del Experimento II. También aquí se trata de la misma palabra, la derivada, sólo que, en el primer caso, era la primera vez que aparecia, mientras que en el segundo, había sido precedida por su forma base. Denominamos $\mathrm{Y}_{1}$ a la primera de estas puntuaciones, $\mathrm{Y}_{2}$ a la segunda $\mathrm{y} W$ a la diferencia.

Así pues, $\mathrm{V}=\mathrm{X}_{1}-\mathrm{X}_{2}$ y $\mathrm{W}=\mathrm{Y}_{1}-\mathrm{Y}_{2}$. (Conviene advertir que aquí, desde el principio, se tomaron conjuntamente los datos de las tres categorías gramaticales, dada la 
semejanza obtenida en el patrón de resultados encontrado en cada una de ellas, tanto en el experimento anterior como en éste.)

Una vez calculadas todas las puntuaciones $\mathrm{V}$ y W, se halló el coeficiente de correlación de Pearson ( $r_{\mathrm{Vw}}$ para comprobar el grado de variación conjunta de las dos distribuciones (la de $\mathrm{V}$ y la de $\mathrm{W}$ ) y ver así cuál era el procedimiento más adecuado para poner a prueba la diferencia de medias entre ambas. Asimismo, se averiguó el nivel de significación de la correlación.

Una vez establecido que se iba a seguir el procedimiento para muestras independientes, 'se efectuó una prueba de homogeneidad de la varianza (Hartley, en Winer, 1971). Una vez determinado si se trataba de muestras independientes o relacionadas y si las varianzas de ambas eran homogéneas o no, se procedió a efectuar una diferencia de medias entre $\mathrm{V}$ y $\mathrm{W}$, determinando su significación estadística.

\section{RESULTADOS}

En primer lugar, volvió a comprobarse una vez más el notable efecto de facilitación por repetición (todas las diferencias de medias pertinentes resultaron altamente significativas: $p<0,001$ ).

Por otro lado, se obtuvo una $r_{\mathrm{vw}}=0,08$ ( $\mathrm{p}>0,05)$, lo cual nos llevó a emplear el procedimiento indicado para muestras independientes en la comparación de medias. Los resultados obtenidos en la prueba de homogeneidad de la varianza pusieron de manifiesto la existencia de diferencias significativas entre las varianzas correspondientes a las dos muestras de estímulos utilizadas $\left(\mathrm{F}_{\mathrm{mix}}=2,23>\mathrm{F}_{2,107 \mathrm{~g} .1}=1,00, \mathrm{p}<\right.$ $0.001)$. Por último, la diferencia de medias calculada (V-W $=9 \mathrm{mseg}$.) no resultó significativa $\left(Z^{\prime}=1.06, \mathrm{p}>0,05\right)$.

\section{DISCUSION}

Los resultados parecen indicar que no existen diferencias significativas entre el efecto de facilitación que una palabra deri- vada produce sobre el reconocimiento de la base y la facilitación que ejerce la palabra base sobre el posterior reconocimiento de una de sus formas derivadas.

Cabría plantearse aquí si es que las representaciones de memoria de ambos tipos de formas verbales (base y derivada) son independientes o si existe algún tipo de relación entre las mismas. Esa relación -caso de que se dé- sería una relación simétrica, ya que, a la luz de los resultados, no parece haber dependencia de una de las representaciones con respecto a la otra. Es decir, el proceso de reconocimiento de una palabra derivada no necesariamente tiene que estar mediado, en un sentido estricto, por el reconocimiento de su forma base, como tampoco el reconocimiento de la forma base estaría mediado por el de la derivada.

Ahora bien, existe un resultado que no permite afirmar que las entradas léxicas correspondientes a una forma base y a sus derivadas carezcan por completo de relación. Nos referimos a la facilitación que produce la derivada sobre el posterior reconocimiento de la base (Experimento I), así como a la que ejerce la presentación previa de la base sobre el reconocimiento de la derivada (Experimento II).

Puede mencionarse aquí la interesante observación de Stanners, Neiser, Hernon y Hall (1979), donde señalan que «representaciones separadas en la memoria léxica no significa necesariamente representaciones independientes» (pág. 405). Según estos autores, cuando un individuo está aprendiendo la forma de pasado de un verbo irregular, debe desarrollarse una nueva representación de memoria que dé cuenta de las diferencias en la pronunciación o deletreo de la forma irregular, pero esta nueva versión va a quedar integrada en una estructura de memoria donde está incluido el verbo base. De este modo, siempre que se accede a la representación de la forma de pasado, se activa también el verbo base, sólo que el nivel de activación será menor que cuando se accede directamente a éste último. En los experimentos de estos autores, los resultados parecen indicar incluso que la activación del verbo base podría ser una fase necesaria en el procesamiento de la forma de 
pasado. Conviene recordar también que esta interpretación se ajusta a la explicación de Forster (1976) sobre el efecto de «priming», que ya veíamos en la Discusión del Experimento I.

No obstante, el diseño de este experimento no es el mejor posible, ya que nos obligó a efectuar una comparación a través de dos muestras distintas de sujetos. Por otra parte, la forma de plantear el experimento no nos había permitido efectuar un análisis de varianza, quizá la prueba más adecuada para determinar la naturaleza de los efectos y relaciones que pretendíamos poner de manifiesto. Por estas dos razones, consideramos pertinente diseñar un nuevo experimento donde fuera posible realizar este tipo de análisis.

\section{EXPERIMENTO III}

Este experimento incluye todas las condiciones experimentales de los dos anteriores (base-base, derivada-base y base-derivada) más una condición de repetición con palabras derivadas (derivada-derivada).

El objetivo que se persigue es determinar el tipo de relación existente entre la representación mental de las formas base y las derivadas. Los resultados conjuntos de los Experimentos I y II indican una relación simétrica entre ambas representaciones. No obstante, decidimos diseñar un nuevo experimento para verificar este fenómeno con una misma muestra de sujetos (distinta de las dos anteriores) e incluyendo una nueva condición que nos permitiera el contrabalanceo adecuado.

Los derivados que se utilizaron en este experimento consistieron únicamente en nombres, dado que se había comprobado previamente que no había diferencias en la actuación de las tres categorías gramaticales. Al no ser condición necesaria que cada palabra base diese lugar a un nombre, un verbo $\mathrm{y}$ un adjetivo derivados, pudimos ejercer un mayor control sobre los estímulos seleccionados, eligiendo terminaciones más variadas para las derivaciones (para este propósito, se utilizó el Reverse Dictionary of the Spanish Language, de Stahl y Scavnicky, 1973).
Otra diferencia importante con respecto a los Experimentos I y II fue que, en este caso, la presentación de los estímulos fue auditiva. La mayor parte de las investigaciones sobre reconocimiento de palabras y procesamiento del lenguaje, en general, se han realizado empleando técnicas de presentación visual. El supuesto de que los resultados de los experimentos visuales puedan generalizarse a la modalidad auditiva no parece carente de fundamento, dado que está claro que ambos modos de procesar el lenguaje están estrechamente relacionados. Por ejemplo, las representaciones grafémicas de las palabras son formas codificadas de su estructura fonológica y el aprendizaje de estas formas normalmente está mediado por el sistema auditivo (Garret; 1978). No obstante, también hay razones para sospechar que existan diferencias entre los mecanismos de procesamiento de cada modalidad, dadas las notables divergencias en la naturaleza de la señal física con la que opera cada sistema (Egido, 1982).

Existe una clara escisión entre los modelos actuales al uso del procesamiento del lenguaje con respecto a esta cuestión. De una parte, los modelos como el que proponen Forster y sus colaboradores (Forster y. Chambers, 1973; Forster y Bednall, 1976; Forster, 1976, 1979, 1981a, 1981b), que defienden la autonomía entre procesos lingüísticos y variables contextuales, asumen solamente diferencias superficiales en el procesamiento entre ambas modalidades. Estos modelos «autonómicos» suponen que la estructura informacional del lenguaje en sí es la que ejerce la principal influencia sobre el procesamiento y, en este sentido, defienden la generalización entre modalidades de procesamiento.

De otro lado, Marslen-Wilson y sus colegas (Marslen-Wilson, 1976, 1980; Marslen-Wilson y Welsh, 1978; Marslen-Wilson y Tyler, 1980; Tyler y Marslen-Wilson, $1981,1982)$ han propuesto un modelo de procesamiento del lenguaje dirigido específicamente a la modalidad auditiva que incorpora los efectos contextuales y pragmáticos a las etapas de acceso léxico y procesamiento sintáctico. En este modelo, las características físicas del input están inextri- 
cablemente ligadas al modo como tiene lugar el procesamiento. (Para una discusión y comparación más detallada de ambos modelos, véase del Viso, 1984, págs. 12-29).

El modelo de Forster se basa en datos obtenidos a partir de la modalidad visual, mientras que el modelo de la «cohorte» de Marslen-Wilson se apoya en un cuerpo de datos que provienen solamente de la modalidad auditiva. Por ello, uno se ve tentado a argumentar que los dos modelos están en conflicto no sólo por sus respectivos puntos de partida teóricos, sino también porque describen sistemas diferentes -el procesador visual del lenguaje y el procesador auditivo-, aunque los resultados conflictivos de las dos modalidades no implican necesariamente diferencias importantes de procesamiento, sino que podrían ser meramente producto de las diferencias que se dan en los detalles más finos y periféricos del mismo.

Así pues - y dado que lo que se pretende, en último término, es dar con un modelo general del procesamiento que tiene lugar entre el análisis del input sensorial (cualquiera que sea su descripción física) y la localización de las entradas léxicas (García-Albea, del Viso y Sánchez-Casas, 1985) - , parece conveniente efectuar comparaciones más sistemáticas entre datos visuales y auditivos para aclarar esta cuestión. Por el momento, disponemos de pocos trabajos experimentales en esta línea. Uno de ellos es el de Egido (1982), que consiste en una réplica, con presentación auditiva, de uno de los experimentos de Bradley (1978). A partir de los resultados obtenidos, la autora concluye que el paralelismo encontrado en el reconocimiento en ambas modalidades constituye un apoyo a la idea de que la estructura del lenguaje ejerce sobre la organización del procesamiento una influencia mucho más importante que la que ejerce la naturaleza física de la señal.

\section{METODO}

Sujetos.. Participaron 40 sujetos (10 hombres y 30 mujeres), estudiantes de 50 de Psicología. Fueron seleccionados aleato- riamente, comprobando que fuesen monolingües. Se les asignó a uno de cuatro grupos experimentales, compuestos por $10 \mathrm{su}$ jetos cada uno. Todos los grupos fueron expuestos a la misma secuencia de estímulos, variando las condiciones experimentales de un grupo a otro.

Materiales. Se seleccionaron 32 palabras (nombres primitivos en singular) que no fuesen ambiguas ni en categoría gramatical ni en significado y que pudieran dar lugar a otros 32 nombres derivados (por ej., carta-cartero; piano-pianista). A partir de la estructura morfológica y ortográfica de los 32 nombres primitivos, se construyó una lista de 32 no-palabras con apariencia de nombres y que pudieran dar lugar a las correspondientes «derivaciones» (por ej., arcia-arciero; redeno-redenista).

Además, se utilizaron 56 elementos de «relleno» que servían para organizar la secuencia de presentación de los estímulos. De estos 56 elementos, 28 eran palabras y 28 , no-palabras. Dentro de las palabras, había 14 nombres primitivos y 14 derivados, independientes unos de otros (por ej., limón, peineta, acento, patinaje). La misma proporción se mantuvo en las no-palabras.

Así, pues, en total, se utilizaron 184 estímulos: 92 palabras y 92 no-palabras. Dentro de las palabras, 64 eran propiamente experimentales y 28 eran de «relleno», al igual que en las no-palabras.

El principal criterio para seleccionar las palabras fue que tuvieran una derivación clara, directa y usual. Debido a que los estímulos se iban a presentar auditivamente, no se midió la longitud por el número de letras - como se había hecho en los experimentos anteriores-, sino por el número de sílabas.

Los estímulos utilizados se presentan en el Apéndice II.

Diseño. A cada uno de los estímulos primitivos se le asignaba aleatoriamente un intervalo entre 7 y 10 . Este determinaba, como en los experimentos anteriores, el número de estímulos que se presentarían entre un "prime» determinado y su «target» correspondiente. Los distintos intervalos $(7 / 8 / 9 / 10)$ aparecían el mismo número de veces. 
Se establecieron las cuatro condiciones experimentales ya mencionadas. La asignación de cada condición a los distintos eștímulos se hizo dividiendo, en primer lugar, la lista original de 32 elementos en cuatro partes de 8 estímulos. A continuación, se asignaron las condiciones experimentales a cada una de las cuatro partes, rotándolas a través de los cuatro grupos de sujetos. De este modo, a todos los sujetos se les presentaban todos los estímulos y todas las condiciones, pero nunca el mismo estímulo en distintas condiciones. Además, todos los estímulos aparecieron bajo las cuatro condiciones, pero nunca para un mismo sujeto o grupo de sujetos.

Los estímulos se distribuyeron en cuatro bloques de 46 elementos cada uno. Dentro de cada bloque, aparecían en un orden cuasi-aleatorio, con las restricciones que ya comentamos con respecto al Experimento I. Dentro de cada grupo de sujetos, el orden de presentación de los bloques fue aleatorio, alternándose entre los sujetos. Antes de comenzar la tarea experimental propiamente dicha, se presentaba un bloque de práctica con 32 estímulos de los mismos tipos que los experimentales para familiarizar al sujeto con la tarea y la situación experimental.

Procedimiento. Los estímulos fueron grabados en cinta magnetofónica por un.hablante femenino. El intervalo entre el final de un estímulo y el comienzo del siguiente fue de 4 segundos. Cada estímulo iba precedido por una señal de aviso para que el sujeto estuviera preparado. Esta señal se presentaba unos 2 segundos antes del estímulo en cuestión.

Se utilizó un reproductor estéreo SANYO (Modelo M-4430) provisto de unos auriculares para el sujeto. El volumen de reproducción se mantuvo constante. Se empleó un aparato que transformaba los impulsos magnéticos grabados en la cinta en señales que ponían en marcha el medidor de TR, de tal forma que éste sólo se ponía en funcionamiento al iniciarse cada nuevo estímulo y se detenía cuando el sujeto pulsaba las llaves de respuesta. Se utilizó además un programador de tiempo de cuatro canales, con las mismas funciones que en los experimentos anteriores.
La tarea fue nuevamente la de decisión léxica. El sujeto recibía las instrucciones grabadas en cinta por el mismo hablante que había grabado los estímulos experimentales.

Tratamiento de los datos. Al igual que en los Experimentos I y II, se eliminaron los datos que constituían errores y los superiores o inferiores a la media en $1,5 \mathrm{~s}_{\mathbf{x}}$.

Se llevó a cabo un análisis por estímulos y otro por sujetos. En cada uno, se realizó primeramente un análisis de varianza, utilizando además el procedimiento de Clark (1973). Cada ANOVA respondía a un diseño factorial $4 \times 2 \times 2 \times 2$, donde el primer factor era "grupo de sujetos» (en el análisis por sujetos) o «lista de estímulos (en el análisis por estímulos). En este último, el segundo factor era «tipo de palabra» (base/derivada); el tercero, «condición experimental» (repetición/crítica); y el cuarto, «posición» (prime/target). En este análisis, había medidas repetidas en los factores tercero y cuarto. En el análisis por sujetos, el segundo factor era «condición experimental»; el tercero, «tipo de palabra»; y el cuarto, «posición», con medidas repetidas solamente en el segundo.

En segundo lugar, se analizaron los resultados en función de los efectos experimentales que nos interesaba estudiar. Para ello, se compararon los TR medios obtenidos bajo cada una de las distintas condiciones en que podía aparecer una palabra, calculándose su significación estadística mediante la «t» de Student. Se analizaron los siguientes efectos: 1) efecto de facilitación por repetición o identidad, en las palabras base y en las derivadas; 2) efecto de facilitación por relación morfológica, tanto de la derivada sobre la base como de ésta sobre la derivada; 3) comparación de los dos efectos anteriores; y 4) comparación de los dos efectos de facilitación por relación morfológica.

\section{RESULTADOS}

En las tablas 3 y 4 , se presentan los TR medios a partir de los cuales se realizaron los ANOVA. 
TABLA 3

Tiempos de reacción medios bajo las distintas condiciones a partir de los cuales se realizó el análisis de varianza por estimulos $(N=32)-E X P$. III-

\begin{tabular}{|c|c|c|c|c|}
\hline \multirow[b]{2}{*}{ Tipo palabra } & \multicolumn{2}{|c|}{ Cond. Repetición } & \multicolumn{2}{|c|}{ Cond. Crítica } \\
\hline & Prime & Target & Prime & Target \\
\hline $\begin{array}{l}\text { Base } \\
\text { Derivada } \ldots \ldots \ldots \ldots \ldots \ldots \ldots \ldots \ldots \ldots \ldots \ldots \ldots \ldots\end{array}$ & $\begin{array}{l}695 \\
816\end{array}$ & $\begin{array}{l}620 \\
715\end{array}$ & $\begin{array}{l}694 \\
824\end{array}$ & $\begin{array}{l}661 \\
774\end{array}$ \\
\hline
\end{tabular}

\section{TABLA 4}

Tiempos de reacción medios bajo las distintas condiciones a partir de los cuales se realizó el análisis de varianza por sujetos $(N=40)-E X P$. III-

\begin{tabular}{|c|c|c|c|c|}
\hline \multirow{2}{*}{ Tipo palabra } & \multicolumn{2}{|c|}{ Cond. Repetición } & \multicolumn{2}{|c|}{ Cond. Crítica } \\
\hline & Prime & Target & Prime & Target \\
\hline 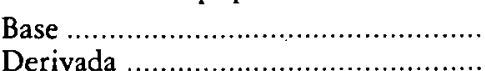 & $\begin{array}{l}703 \\
826\end{array}$ & $\begin{array}{l}618 \\
714\end{array}$ & $\begin{array}{l}692 \\
836\end{array}$ & $\begin{array}{l}666^{\circ} \\
777\end{array}$ \\
\hline
\end{tabular}

En el ANOVA por estímulos, resultaron significativos los factores «tipo de palabra» $(\mathbf{F}(1,56)=32,25, \mathrm{p}<0,001)$, «condición experimental» $(\mathrm{F}(1.168)=10,66, \mathrm{p}<0,001)$ y "posición» $(\mathrm{F}(1,168)=6,14, \mathrm{p}<0,01)$, así como la interacción «condición experimental x posición» $(\mathrm{F}(1.168)=7,99, \mathrm{p}<0,001)$. Estos resultados indican, en primer lugar, que, en general, las palabras base se reconocen antes (el TR es menor) que las derivadas. En segundo lugar, que, en general, el TR es menor en la condición de repetición que en la crítica, independientemente de si las palabras son base o son derivadas y de si están en posición de "prime» o de «target». En tercer lugar, que los «targets» se reconocen antes que los «primes», es decir, que se dan efectos de facilitación por presentación previa, independientemente de la condición experimental y del tipo de palabra. Finalmente, la interacción «condición $\mathrm{x}$ posición» indica que la ventaja (TR menores) de la condición de repetición sobre la crítica se da únicamente con respecto a los «targets», siendo esta ventaja del «target» sobre el «prime» mayor en la condición de repetición.
En el ANOVA por sujetos, interesa señalar la significación alcanzada por los factores «condición experimental» (F (1.252) $=50,4, \mathrm{p}<0,001)$, «tipo de palabra» (F $(1.252)=941,8, \mathrm{p}<0,001), \mathrm{y}$ «posición» $(\mathrm{F}$ $(1.252)=335,2, \mathbf{p}<0,001)$, así como por las interacciones «condición experimental $\mathbf{x}$ tipo de palabra» $(\mathrm{F}(1.252)=5,1, \mathrm{p}<0,01)$, «condición experimental $x$ posición» ( $F$ $(1.252)=53,8, \mathrm{p}<0,001)$ y «tipo de palabra $\mathrm{x}$ posición» $(\mathrm{F}(1.252)=15,2, \mathrm{p}<0,001)$. Lo que añaden estos resultados con respecto a los obtenidos en el análisis por estímulos es lo siguiente: en primer lugar, que la ventaja de la condición de repetición sobre la crítica es mayor en las palabras derivadas que en las bases (interacción «condición $\mathbf{x}$ tipo de palabra»); y en segundo lugar, que la diferencia entre los TR correspondientes a la base y a la derivada disminuye cuando ambas aparecen como «target». Asimismo, la diferencia entre los TR medios correspondientes a los «primes» y a los «targets» es menor en las palabras base que en las derivadas.

Al combinar los resultados de los análisis por estímulos y por sujetos mediante el 
procedimiento de Clark, se obtuvieron efectos significativos de los tres factores principales: «condición experimental» $(\min \mathrm{F}$ $(1.239)=8,80, \mathrm{p}<0,01)$, «tipo de palabra» $\left(\min \mathrm{F}^{\prime}(1,59)=31,18, \mathrm{p}<0,001\right)$ y $\ll$ posición» $\left(\min \mathrm{F}^{\prime}(1.174)=6,03, \mathrm{p}<0,05\right)$. Sola- mente resultó significativa la interacción «condición experimental x posición» (min $\left.F^{\prime}(1.218)=6,96, p<0,01\right)$.

Por otra parte, en las tablas 5 y 6 se presentan los TR medios obtenidos en cada una de las distintas condiciones bajo las que

\section{TABLA 5}

Tiempos de reacción medios en cada una de las distintas condiciones y efectos de facilitación obtenidos (analisis por estimulos) -EXP. III-

\begin{tabular}{ccccccc}
\hline & Rep. & Base & Crit. & Rep. & Deriv. & Crít. \\
\hline PRIME & & 695 & & & 820 & \\
\hline TARGET & 620 & 661 & 714 & 774 \\
\hline \hline $\begin{array}{c}\text { EFECTOS DE } \\
\text { FACILITACION }\end{array}$ & 75 & & 34 & 106 & 46 \\
\hline
\end{tabular}

TABLA 6

Tiempos de reacción medios en cada una de las distintas condiciones y efectos de facilitación obtenidos (análisis por sujetos) -EXP. III-

\begin{tabular}{|c|c|c|c|c|}
\hline & Rep. & Crít. & Rep. & Crit. \\
\hline PRIME & \multicolumn{2}{|c|}{698} & \multicolumn{2}{|c|}{831} \\
\hline TARGET & 618 & 666 & 714 & 777 \\
\hline $\begin{array}{l}\text { EFECTOS DE } \\
\text { FACILITACION }\end{array}$ & 80 & 32 & 117 & 54 \\
\hline
\end{tabular}

podían aparecer los estímulos, así como los efectos de facilitación obtenidos en cada caso, y entre los que establecimos las comparaciones oportunas.

Los resultados pusieron de manifiesto nuevamente un claro efecto de facilitación por repetición, tanto en las palabras base como en las derivadas, que ya venía indicado por la significación alcanzada en el ANOVA por el factor principal «posición». En segundo lugar, se encontraron también efectos de facilitación por relación morfo- lógica, tanto de la derivada sobre la base $(t=$ 3,01 , g. $1 .=31, p<0,01$, en el análisis por estímulos y $\mathrm{t}=5,4, \mathrm{~g} .1 .=39, \mathrm{p}<0,01$, en el análisis por sujetos), como de la base sobre la derivada $(t=2,99$, g. $1 .=31, p<0,01$, en el análisis por estímulos y $\mathrm{t}=6,5, \mathrm{~g} .1$. = $39, \mathrm{p}<0,01$, en el análisis por sujetos). En tercer lugar, se observó que el efecto de facilitación por relación morfológica era menor que el de facilitación por repetición, en los dos tipos de palabras; este resultado venía indicado por la significación alcanzada 
en el ANOVA por la interacción «condición experimental x posición». Por último, al comparar los dos efectos de facilitación por relación morfológica, se encontraron divergencias entre el análisis por estímulos y por sujetos. En el primero, resultaron equivalentes ambos efectos de facilitación $(\mathrm{t}=-0,61, \mathrm{~g} .1 .=31, \mathrm{p}>0,05)$. En el análisis por sujetos, sin embargo, se encontró una facilitación mayor de las bases sobre las derivadas que al contrario $(t=-2,09$, g. 1. $=39, \mathrm{p}<0,05)$.

\section{DISCUSION}

El objetivo principal de este experimento era estudiar de un modo más controlado la relación entre la representación léxica de las palabras base y derivadas, a través del paradigma de "priming".

Los resultados indican, por un lado, que las palabras base se reconocen antes que las derivadas. Este dato pone de manifiesto el efecto de la frecuencia de uso (en general, mayor en las palabras base) y de la longitud (mayor en las derivadas) sobre el reconocimiento de palabras. Ahora bien, otro de los resultados, la interacción «tipo de palabra x posición» en el ANOVA por sujetos, indica que esta diferencia entre los TR correspondientes a las palabras base y las correspondientes a las derivadas disminuye cuando ambas se presentan como «target», es decir, cuando han sido precedidas por otra palabra (sea la misma, sea una relacionada). Este dato es consistente con el efecto de atenuación de la frecuencia, según el cual parece que los efectos de facilitación por repetición en tareas de decisión léxica son mayores para las palabras de baja frecuencia que para las de frecuencias más altas (Forster y Davis, 1983). Aplicando este efecto al caso que nos ocupa, cabría pensar que las palabras derivadas (en general, con una frecuencia de uso menor que las base) se han visto más afectadas por los efectos del «priming».

Ahora bien, Forster y Davis (1983) atribuyen este efecto de atenuación de la frecuencia a factores episódicos de recencia de la presentación de una palabra, más allá de los factores puramente léxicos. En un intento de minimizar la influencia de los factores episódicos para aislar las propiedades del procesamiento léxico en sí, estos autores proponen que en los experimentos donde se utilice el paradigma de «priming», debe presentarse una máscara para el «prime» y, por otro lado, que no debe hacerse al sujeto responder al "prime» sino solamente al «target». Ambas medidas se adoptan con objeto de minimizar la experiencia consciente del sujeto con respecto al «prime» y, por tanto, minimizar la contribución de los factores episódicos. Los autores encuentran que ambas medidas son eficaces para este propósito. En nuestros experimentos, no hemos aplicado ninguna de ellas y podría argumentarse que nuestros resultados no permiten establecer conclusiones acerca del funcionamiento y la organización del léxico, ya que estarían contaminados por los factores episódicos. Sin embargo, hay un dato que nos permite salir al paso de estas críticas. Nos referimos a la inclusión en nuestros experimentos de las que hemos denominado condiciones críticas, donde el «target» no va precedido exactamente por sí mismo, sino por otra palabra distinta relacionada morfológicamente. En este caso, no puede hablarse ya de la influencia exclusiva de los factores episódicos, puesto que no se trata de la misma palabra presentada en dos ocasiones, sino de dos palabras distintas. Pensamos, por esta razón, que los efectos aquí observados están determinados principalmente por el componente léxico.

Por otro lado, hemos observado que, en general, los «targets» se reconocen antes que los «primes», lo cual viene a confirmar la existencia de un efecto de facilitación por presentación previa, ya sea de la misma palabra o de otra relacionada. Dentro de esto, nuestros resultados indican que el efecto de facilitación por repetición es superior al de facilitación por relación morfológica. Los «primes», lógicamente, no se ven afectados por la condición experimental en la que aparecen. Donde sí influye la condición es sobre los «targets», siendo menor el TR de éstos en la condición de repetición que en la crítica.

Habiéndose probado la existencia de un 
efecto de facilitación por la presentación previa de una palabra relacionada morfológicamente, pasaremos a comentar los efectos de facilitación que produce la base sobre la derivada y ésta sobre la base. Solamente en el análisis por sujetos se encontró un efecto de facilitación significativamente mayor de la base sobre la derivada; es decir, parece que cuando la base aparece como «prime» de la derivada, la facilitación que se obtiene es mayor que en el caso de que la derivada se presente como "prime» de la base. No obstante, el análisis por estímulos parece poner de manifiesto un efecto equivalente en ambos casos. Dada esta divergencia entre los dos análisis, los resultados de este experimento - tomados aisladamente- no permiten ser concluyentes con respecto a este punto. La interpretación conjunta de los resultados de éste y de los dos experimentos anteriores se presentará en la discusión general.

En otro orden de cosas, el hecho de haber encontrado los mismos efectos de «priming» que en los experimentos anteriores indica que es posible un cierto grado de generalización de una modalidad sensorial a otra (visual-auditiva). Esto parece apoyar la idea de que ambos modos de procesar el lenguaje están relacionados a nivel central ( $\mathrm{Ga}$ rret, 1978), si bien no excluimos la posibilidad de que puedan darse diferencias en los niveles más periféricos del procesamiento.

\section{DISCUSION GENERAL}

El objetivo principal de este trabajo ha sido contribuir a un mayor conocimiento del papel que desempeñan las variables morfológicas en la organización del léxico interno $y$, por consiguiente, en las estrategias que emplean los sujetos para acceder a la representación mental de las palabras.

En general, nuestros resultados apoyan lo que cabría esperar si se asume una representación común para las palabras relacionadas morfológicamente, a saber, una disminución significativa del TR al presentar una palabra precedida por otra relacionada. Hemos encontrado un efecto de facilitación por repetición y, lo que es más relevante, un claro efecto de facilitación por relación morfológica, es decir, que el hecho de presentar previamente una palabra relacionada morfológicamente con la que aparecerá tras un cierto intervalo temporal hace que ésta segunda se reconozca más rápidamente que cuando no va precedida por la primera. Este efecto de facilitación por relación morfológica se ha observado tanto cuando la palabra que se presenta como «prime» es una derivada de la que aparecerá como «target» como en el caso contrario (base como "prime» y derivada como «target»). Los resultados de los experimentos I y II, tomados conjuntamente, asi como los del III sugieren la existencia de una entrada léxica común para las formas base y sus derivadas.

Centrándonos en el efecto de facilitación que produce una palabra derivada sobre el posterior reconocimiento de su base correspondiente, esto parece apoyar la hipótesis de que el sujeto efectúa una descomposición morfológica de la palabra derivada en «raíz + sufijo» y se centra en la primera para acceder al léxico. Cuando se presenta después la palabra base, va a tener lugar un reconocimiento más rápido, ya que su entrada léxica ha sido accedida previamente al presentarse la forma derivada y tener lugar la descomposición. Nuestros resultados y esta explicación de los mismos son claramente consistentes con el modelo de acceso que proponen Taft y Forster $(1975,1976)$ y Taft (1979b, 1981).

De forma análoga, la facilitación que produce la base sobre el reconocimiento ulterior de una forma derivada también apoya esta hipótesis. En este caso, se presenta la forma base y, durante el proceso de reconocimiento, el sujeto accederá a la entrada léxica correspondiente. Al presentarse después la derivada, el sujeto llevará a cabo la descomposición de la misma, eliminando los sufijos y centrándose en la forma base para proceder a la búsqueda en el léxico; es decir, el sujeto buscará la entrada correspondiente a la forma base. Teniendo en cuenta que ya se ha accedido a dicha entrada recientemente (cuando se presentó la base), se asume que ésta se encontrará más 
disponible, produciéndose los efectos de facilitación debido precisamente a esta mayor disponibilidad.

Es de destacar que el efecto de facilitación por presentación previa se ha constatado tanto mediante presentación visual de los estímulos como mediante presentación auditiva. Esto nos permite conferir una mayor generalidad a nuestros resultados. Además, constituye un apoyo a la idea de que las propiedades fundamentales de ambos sistemas de procesar el lenguaje serían muy similares a nivel central.

Convien̨e señalar que nuestros resultados coinciden, en general, con los obtenidos en otros idiomas, fundamentalmente en inglés, idioma en que se han realizado la mayoría de los trabajos pertenecientes a este campo de estudio. Este hecho constituye un sólido apoyo a la validación translingüística de los resultados concernientes a la organización del léxico. Asimismo, queremos subrayar la convergencia de nuestros resultados con los obtenidos en otras áreas psicolingüísticas (producción, adquisición y patología del lenguaje).

A modo de conclusiones generales, se pueden entresacar las siguientes:

Nuestro trabajo ha puesto de manifiesto, en líneas generales, la realidad psicológica de las relaciones morfológicas. Pensamos que las variables morfológicas no son solamente instrumentos que utiliza el lingüista en su tarea, sino que desempeñan un papel importante en la organización de las palabras en el sistema cognitivo del hablanteoyente.

Nuestros resultados no son concluyentes con respecto a la forma precisa de representación léxica. No hemos planteado nues- tros experimentos como experimentos críticos que nos permitieran decidirnos por un modelo de representación léxica. en contra de otros. En principio, los resultados serían compatibles con las dos hipótesis que hemos expuesto (representación común vs. representaciones separadas). Sin embargo, parecen estar más en consonancia con la primera, entendiendo que dicha representación común tendría un carácter eminentemente abstracto, representativo de lo que hemos considerado como forma base, así como de las formas con sufijos.

Aun admitiendo la segunda hipótesis, los resultados indican que habría que constreñirla en función de las relaciones de dependencia que habría que postular entre esas entradas separadas (recordemos, de nuevo, la observación de Stanners et al., 1979, de que representaciones separadas en la memoria léxica no significa necesariamente representaciones independientes).

A propósito de esta discusión, Bradley (1979) concluye que es posible la existencia de más de un formato de representación léxica. Parece que una variable importante consiste en que al añadir el sufijo derivativo, se cambie o no la sílaba acentuada de la forma base. A este respecto, y teniendo en cuenta la conveniencia de diseñar experimentos críticos, pensamos que quizás sería interesante replicar estos experimentos distinguiendo dos o más tipos de palabras, según su mayor o menor semejanza con la forma base, en función de variables tales como, por ejemplo, la posición del acento. Por otra parte sería deseable comprobar si se obtiene este mismo patrón de resultados con otro tipo de tareas y bajo distintas circunstancias experimentales. 


\section{APENDICE I}

Estimulos utilizados en los Experimentos I y II.
A. Estímulos experimentales

\section{PALABRAS}

Base

(Nombres)

\begin{tabular}{|c|c|c|c|}
\hline & Nombres & $\begin{array}{l}\text { Derivadas } \\
\text { Verbos }\end{array}$ & Adjetivos \\
\hline $\begin{array}{l}\text { vida } \\
\text { bumo }\end{array}$ & $\begin{array}{l}\text { vitalismo } \\
\text { bumareda }\end{array}$ & $\begin{array}{l}\text { vitalizar } \\
\text { bumear }\end{array}$ & $\begin{array}{l}\text { vital } \\
\text { humeante }\end{array}$ \\
\hline $\operatorname{arco}$ & arquero & arquear & arqueado \\
\hline $\begin{array}{l}\text { toro } \\
\text { flor }\end{array}$ & $\begin{array}{l}\text { torero } \\
\text { florero }\end{array}$ & $\begin{array}{l}\text { torear } \\
\text { florecer }\end{array}$ & $\begin{array}{l}\text { taurino } \\
\text { florido }\end{array}$ \\
\hline mano & manubrio & manejar & manual \\
\hline ánimo & animador & animar & animoso \\
\hline polvo & polvareda & pulverizar & polvoriento \\
\hline color & colorido & colorear & colorista \\
\hline señor & señorío & señorear & señorial \\
\hline fruta & frutero & fructificar & frutal \\
\hline valor & valentia & valorar & valioso \\
\hline golpe & golpista & golpear & golpeado \\
\hline ansia & ansiedad & ansiar & ansioso \\
\hline tapiz & tapicero & tapizar & tapizado \\
\hline grado & graduación & graduar & gradual \\
\hline favor & favoritismo & favorecer & favorable \\
\hline verano & veraneante & veranear & veraniego \\
\hline poesia & poeta & poetizar & poético \\
\hline médico & medicina & medicar & medicinal \\
\hline azúcar & azucarero & azucarar & azucarado \\
\hline cabeza & cabecera & cabecear & cabezudo \\
\hline número & numerador & numerar & numeroso \\
\hline sombra & sombrilla & sombrear & sombrio \\
\hline nación & nacionalidad & nacionalizar & nacional \\
\hline imagen & imaginería & imaginar & imaginativo \\
\hline rastro & rastrojo & rastrear & rastreado \\
\hline acento & acentuación & acentuar & acentuado \\
\hline limite & limitación & limitar & limitrofe \\
\hline cristal & cristalera & cistalizar & cristalino \\
\hline fórmula & formulario & formular & formulable \\
\hline almacén & almacenaje & almacenar & almacenado \\
\hline ilusión & ilusionista & ilusionar & ilusorio \\
\hline buesped & bospedaje & bospedar & bospedado \\
\hline perfume & perfumeria & perfumar & perfumado \\
\hline
\end{tabular}


NO-PALABRAS

\begin{tabular}{|c|c|c|c|}
\hline rina & rinalismo & rinalizar & rinal \\
\hline tulo & tulareda & tulear & tuleante \\
\hline enco & enquero & enquear & enqueado \\
\hline loso & losero & losear & lausino \\
\hline glor & glorero & glorecer & glorido \\
\hline pano & panubrio & panejar & panual \\
\hline glafa & glafero & glafear & glafeado \\
\hline ónito & onitador & onitar & onitoso \\
\hline colso & colsareda & colserizar & colsoriento \\
\hline nulor & nulorido & nulorear & nulorista \\
\hline lañor & lañorío & lañorear & lañorial \\
\hline crota & crotero & crotificar & crotal \\
\hline gelor & gelentía & gelorar & gelioso \\
\hline salpe & salpista & salpear & salpeado \\
\hline arfia & arfiedad & arfiar & arfioso \\
\hline roliz & rolicero & rolizar & rolizado \\
\hline brano & branuación & branuar & branual \\
\hline nevor & nevoritismo & nevorecer & nevorable \\
\hline sapano & sapaneante & sapanear & sapaniego \\
\hline caesía & caeta & caetizar & caético \\
\hline nólico & nolicina & nolicar & nolicinal \\
\hline núcaro & nucarero & nucarar & nucarado \\
\hline lotiza & loticera & loticear & lotizudo \\
\hline sánero & sanerador & sanerar & saneroso \\
\hline tembra & tembrilla & tembrear & tembrío \\
\hline gesión & gesionalidad & gesionalizar & gesional \\
\hline elagen & elaginería & elaginar & elaginativo \\
\hline pistro & pistrojo & pistrear & pistreado \\
\hline abelto & abeltuación & abeltuar & abeltuado \\
\hline nápite & napitación & napitar & napítrofe \\
\hline brispol & brispolera & brispolizar & brispolino \\
\hline párfula & parfulario & parfular & parfulable \\
\hline celatén & celatenaje & celatenar & celatenado \\
\hline onusión & onusionista & onusionar & onusorio \\
\hline cuertal & cortalaje & cortalar & cortalado \\
\hline larmufe & larmufería & larmufar & larmufado \\
\hline
\end{tabular}

\section{B. Estímulos de «relleno»}

PALABRAS: miel, miedo, salud, sangre, tiempo, espalda, letrero, acogedor, fatigado, manchado, luminoso, perdonar, marítimo, seguidor, originar, comunidad, curiosear, oscurecer, ligamento, sirviente, felicitar, testificar, fantástico, nocturnidad.

NO-PALABRAS: sael, foeno, pelud, tungre, ruenco, sepolda, sopiero, atuledor, forelado, terchado, pabiloso, garnipar, dorítimo, matridor, calenizar, semoridad, bucanear, lemurecer, padocento, tarciente, dremonar, pastonicar, ceprástico, fastenidad 


\section{APENDICE II}

Estímulos utilizados en el Experimento III.

\section{A. Estímulos experimentales}

\section{PALABRAS}

Base

llave

carta

jabón

silla

señor

granja

árbol

timón

piedra

trauma

hierba

viento

cristal

fiesta

sastre

tapiz

piano

átomo

paella

máquina

moneda

arena

médico

álamo

novela

correa

mercado

ceniza

deporte

gallina

montaña

castaña

\section{Derivadas}

llavero

cartero

jabonera

sillería

señorío

granjero

arboleda

timonel

pedregal

traumatismo

herbolario

ventisca

cristalera

festival

sastrería

tapicero

pianista

atomismo

paellera

maquinista

monedero

arenal

medicina

alameda

novelista

correaje

mercader

cenicero

deportista

gallinero

montañismo

castañera
Base

colso

arcia

lesón

crota

lañor

glafa

névol

cimán

brena

crasma

tierpa

rendo

brispol

nasta

sarpo

roliz

redeno

párfula

lotiza

núcaro

colida

adina

nólico

nápile

delera

balea

mosado

caeta

sapano

lertana

salena

tolaña
NO-PALABRAS

Derivadas

colsero

arciero

lesonera

crotería

lañorío

glafero

nevoleda

cimanel

brenegal

crasmatismo

terpolario

rendisca

brispolera

nastival

sarpería

rolicero

redenista

parfulismo

loticera

nucarista

colidero

adinal

nolicina

napileda

delerista

baleaje

mosader

caetero

sapanista

lertanero

salenismo

tolañera

\section{B. Estímulos de «relleno»}

PALABRAS: limón, tiempo, rastro, lluvia, violín, fruta, puerta, espalda, hermano, límite, acento, espuma, testigo, almacén, peineta, robledal, bailarín, plumaje, dentista, sirviente, vivienda, cerradura, historiador, fabricante, patinaje, macetero, territorio, rosaleda.

NO.PALABRAS: dulón, ruenco, pistro, dolia, selán, breta, merta, sepolda, socano, gálite, abelto, celuma, fastego, celatén, caneta, ganedal, polatín, fantaje, tembrista, tarciente, feranda, calenura, limufador, josalante, copelaje, talenero, opilorio, geraleda. 


\section{Resumen}

El objetivo principal de esta investigación es el de contribusi a un mayor conocimiento del papel que desempeña la estructura morfológica de las palabras en la organización del léxico interno y, por consiguiente, en las estrategias que emplean los sujetos para acceder a la representación mental de las palabras. Más en concreto, se trata de ver cual es la relación existente entre la representación de la forma base de una palabra (por ej., flor) y la de formas derivadas de la misma (por ej., florero, florecer, florido) de cara a una posible simplificación del inventario mental de entradas léxicas.

Se han realizado tres experimentos, basados en una tarea de decisión léxica y en la utilización de la técnica de "priming" o presentación previa de un supuesto elemento facilitador del reconocimiento de cada estimulo. Se midió la latencia de respuesta para clasificar los estimulos como palabras o no-palabras del idioma. Los dos primeros experimentos se efectuaron bajo la modalidad visual de reconocimiento y el tercero, bajo la modalidad auditiva. En todos los casos, las comparaciones relevantes se establecen entre los TRs correspondientes a una misma palabra en condiciones experimentales diferentes: según vaya precedida por si misma, o por una relacionada morfológicamente, o cuando no vaya precedida ni por si misma ni por otra relacionada (condición de control).

Los resultados obtenidos bajo las dos modalidades perceptivas son coincidentes y ponen de manifiesto que: 1) se da un efecto de facilitación en el reconocimiento, tanto al presentar una palabra precedida por si misma (repetición) como al presentarla precedida por otra relacionada morfológicamente; 2) el efecto de facilitación por repetición es. significativamente superior al de facilitación por relación morfológica; y 3) dentro de este último no aparecen diferencias significativas entre la facilitación de la forma base por la derivada y la de ésta por aquélla.

Estos resultados se interpretan a la luz de los distintos modelos propuestos acerca de la representación lé. xica. Sin que sean cruciales para decidirse por uno de ellos en contra de los otros, si que parecen apoyar la existencia de un código de representación abstracto que aglutina familias de palabras relacionadas morfológicamente en torno a una misma entrada léxica. En cualquier caso, y aun suponiendo que bubiera entradas léxicas distintas para cada forma particular, sería preciso postular algün mecanismo adicional que diera cuenta del papel que desempeña la información morfológica en la organización general del léxico interno.

\section{Abstract}

The main goal of this research bas been to elucidate the role played by morphological structure in the organization of the internal lexicon and, for that matter, in the strategies used to access word mental representations. In particular, this paper is concerned with the relationship between the representation of base forms in Spanish (e. g., flor) and that of their derived counterparts (e. g., florero, florecer, florido), in order to ascertain the possible simplification of the inventory of lexical entries.

Three experiments were performed, based on a lexical decision task and on the use of a word priming technique. Response latencies for word/nonword classification were measured. Experiments 1 and 2 used visual stimuli, whereas experiment 3 used auditory stimuli. In all cases, the relevant comparisons were done between $R T$ s corresponding to the same item in different conditions of presentation: preceded by itself (repetition), preceded by a morphologically related word, or without any prime (control).

Results obtained under both perceptual modalities show the same pattern: 1) there is a facilitatory effect in recognition (lower RTs) either in the repetition condition or with a previous presentation of a morphologically related word; 2) the repetition effect is, in any event, higher than the facilitatory effect due to morpbological relation; 3) within the facilitation by morphological relation, there are not significant differences between the base for priming over the derived form and the priming of the latter over the former.

These results are considered with respect to the available models for lexical representation. They are not crucial in order to decide for one of them against the others, although they seem to support an abstract code of representation which includes under the same lexical entry a whole family of morphologically related words. Even in the case of separated lexical entries for each particular form, some otber mechanism ought to be postulated in order to account for the role played by morphological information in the general organization of the internal lexicon. 


\section{Referencias}

BLANK, M. A. y Foss, D. J.: (1978): «Semantic facilitation and lexical access during sentence processing». Memory and Cognition, 6, 644-652.

BRADLEY, D. C.: (1978): Computational distinctions of vocabulary type. Tesis doctoral no publicada, M.I.T.

- (1979): «Lexical representation of derivational relation», en M. Aronoff y M. L. Kean (Eds.), Juncture. Cambridge, Mass.: M. I. T. Press.

Carroll, M., y KirSner, K.: (1982): «Context and repetition effects in lexical decision and recognition memory». Journal of Verbal Learning and Verbal Bebavior, 21, 55-69.

ClaRK, H. H.: (1973): «The language-as-a-fixed-effect fallacy: A critique of language statistics in psychological research». Journal of Verbal Learning and Verbal Behavior, 12, 335-359.

Collins, A. M., y LOFTUS, E. F.: (1975): «A spreading activation theory of semantic processing». Psychological Review, 82, 407-428.

EGIDO, C.: (1982): Frequency effecrs in auditory lexical decision. Manuscrito no publicado, M.I.T.

FISCHLER, I.: (1977): «Semantic facilitation without association in a lexical decision task.» Memory and Cognition, 5, 335-339.

ForbaCH, G. B.; STANNERS, R. F., y HOCHHAUS, L.: (1974): «Repetition and practice effects in a lexical decision task». Memory and Cognition, 2, 337-339.

Forster, K. I.: (1976): «Accessing the mental lexicon». en E. W. Walker y R. J. Wales (Eds.), New approaches to language mechanisms. Amsterdam: North Holland.

- (1979): «Levels of processing and the structure of the language processor»., en W. Cooper y E. W. Walker (Eds.), Sentence processing: Psycholinguistic studies presented to Merill Garrett. Hillsdale, N. J.: L. E. A.

- (1981a): Frequency blocking and lexical access: One mental lexicon or two? Journal of Verbal Learning and Verbal Behavior, 20, 190-203.

- (1981b): Priming and the effects of sentence and lexical contexts on naming time: Evidence for autonomous lexical processing. Quarterly Journal of Experimental Psychology, 33A, 465-495.

FORSTER, K. I., y BEDNALL, E. S.: (1976): Terminating and exhaustive search in lexical access. Memory and Cog. nition, 4, 53-61.

ForsteR, K. I., y CHAMBERS, S. M. (1973): «Lexical access and naming time». Journal of Verbal Learning and Verbal Behavior, 12, 627-635.

FORSTER, K. I., y DAVIS, C.: (1983): Repetition priming and frequency attenuation in lexical access. Manuscrito no publicado. Monash Universityu.

Foss, D. J. (1982): «A discourse on semantic priming». Cognitive Psychology, 14, 590-607.

GARCIA-AlbEA, J. E. (1980): «Variables estructurales en el reconocimiento visual de palabras», Informes del Departamento de Psicología General (Univ. Complutense), 3/6, 3-22.

- (1984): Syllabic structure and stress in the segmentation of the visual input for word recognition in Spanish. Comunicación presentada en la European Psycholinguistic Association Workshop, "Cross-Linguistic Studies of Morpho-Phonological Processing». París.

Garcia-Albea, J. E., DEL VISO, S., y Sanchez-Casas, R. M. (1985): La percepción del lenguaje: cuestiones metodológicas relacionadas con el reconocimientos visual y auditivo de palabras. Comunicación presentada en el I Congreso Internacional de Psicolingüística Ajplicada. Barcelona.

GARRETT, M. F. (1978): «Word and sentence perception», en R. Held, H. W. Liebowitz y H. L. Teuber (Eds.), Handbook of Sensory Physiology. Vol. VIII: Perception. Berlín: Springer-Verlag.

HOLYOAK, K. J.; GLASS, A. L., y MAH, W. A. (1976): «Morphological structure and semantic retrieval». Journal of Verbal learning and Verbal Bebavior, 15, 235-247.

MANELIS, L., y THARP, D. (1977): The processing of affixed words. Memory and Cognition, 5, 690-695.

MARSLEN-WILSON, W. D. (1976): «Linguistic descriptions and psychological assumptions in the study of sentence perception», en R. J. Wales y E. Walker (Eds.), Approaches to language mechanisms. A collection of psycholinguistic studies. Amsterdam: North Holland.

- (1980): «Speech understanding as a psychological process» en T. C. Simon (Ed.), Spoken language generation and understanding. Dordrecht: Reidel.

MARSLEN-WILSON, W. D., Y TYLeR, L. K. (1980): «The temporal structure of language understanding». Cognition, $8,1-71$.

MARSLEN-WIISON, W. D., y WELSH, A. (1978): «Processing interactions and lexical access during word recognition in continuous speech». Cognitive Psychology, 10, 29-63.

MEYER, D. E., y SCHVANEVELDT, R. W. (1971): «Facilitation in recognizing pairs of words: Evidence of a de- pendence between retrieval operationsm. Journal of Experimental Psychology, 90, 227-234.

- (1976): «Meaning, memory structure, and mental processes». Science, 192, 27-33.

MEYER, D. E.; SChVANEVELDT, R. W., y RUdDY, M. (1972): Activation of lexical memory. Trabajo presentado en la reunión anual de la Psychonomic Science. San Luis.

MORTON, J. (1969): Interaction of information in word recognition. Psychological Review, 76, 165-178.

Rubin, G. S., BeCKer, C. A. y Freeman, R. H. (1979): Morphological structure and its effect on visual word recognition. Journal of Verbal Learning and Verbal Behavior, 18, 757-767. 


\section{Estudios}

SCarborough, D. L., Cortese, C. y Scarborough, H. S. (1977): Frequency and repetition effects in lexical memory. Journal of Experimental Psychology: Human Perception and Performance, 3, 1-17.

SCHVANEveldt, R. W. y MeYER, D.E. (1973): Retrieval and comparison processes in semantic memory. En S. Kornblum (Ed.), Attention and Performance IV. New York: Academic Press.

Stahl, F. y SCavnicky, G. (1973): A Reverse Dictionary of the Spanish Language. University of Illinois Press.

Stanners, R. F., Neiser, J. J., HeRnon, W. P. y Hall, R. (1979): Memory representation for morphologically related words. Journal of Verbal Learning and Verbal Behavior, 18, 399-412.

StANners, R. F., Neiser, J. J y PAinton, S. (1979): Memory representation for prefixed words. Joumal of Verbal Learning and Verbal Behavior, 18, 733-743.

TAFT, M. (1979): Lexical access via an orthographic code: The Basic Orthographic Syllabic Structure (BOSS). Journal of Verbal Learning and Verbal Behavior, 18, 21-39. (a)

- (1979). Recognition of affixed words and the word frequency effect. Memory and Cognition, 7, 263-272. (b)

- (1981): Prefix stripping revisited. Journal of Verbal Learning and Verbal Behavior, 20, 289-297.

TAFT, M. y FORSTER, K. I. (1975): Lexical storage and retrieval of prefixed words. Journal of Verbal Learning and Verbal Behavior, 14, 638-647.

- (1976): Lexical storage and retrieval of polymorphemic and polysyllabic words. Joumal of Verbal Learning and Verbal Behavior, 15, 607-620.

TYLER, L. K. y MARSLEN-WLSON, W. D. (1981): Quick on the uptake. New Scientist, 89, 608-609.

- (1982): Speech comprehension processes. En J. Mehler, E. C. Walker y M. F. Garrett (Eds.), Perspectives on mental representation. Hillsdale, N. J.: L. E. A.

del VISO, S. (1984): El papel de las variables morfológicas en la organización del léxico interno. Memoria de Licenciatura no publicada. Universidad Complutense de Madrid.

del VISO, S. y GARCIA-ALBEA, J. E. (1985): Variables morfológicas en el procesamiento del lenguaje. Estudios de Psicologia, ne 19/20, 195-214.

WINER, B. J. (1971): Statistical Principles in Experimental Design. New York: McGraw-Hill. 